\title{
T-Phantom: a NeW Phantom Design FOR NEUROSURGICAL ROBOTICS
}

\author{
Marko Švaco, Bojan Jerbić, Ivan Stiperski, Domagoj Dlaka, \\ Josip Vidaković, Bojan Šekoranja \& Filip Šuligoj
}
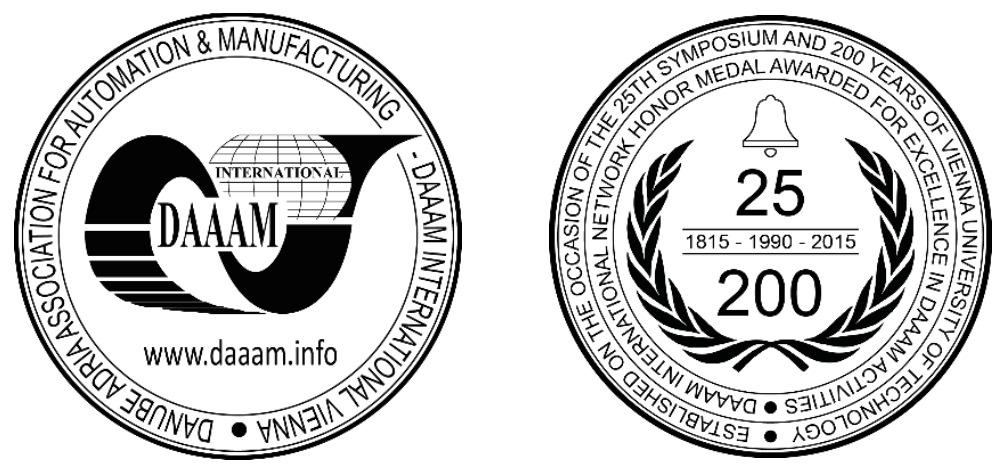

This Publication has to be referred as: Svaco, M[arko]; Jerbic, B[ojan]; Stiperski, I[van]; Dlaka, D[omagoj]; Vidakovic, J[osip] \& Sekoranja, B[ojan] (2016). T-Phantom: a New Phantom Design for Neurosurgical Robotics, Proceedings of the 27th DAAAM International Symposium, pp.0266-0270, B. Katalinic (Ed.), Published by DAAAM International, ISBN 978-3-902734-08-2, ISSN 1726-9679, Vienna, Austria

DOI: $10.2507 / 27$ th.daaam.proceedings.039

\begin{abstract}
In this paper we propose a novel phantom design for measuring application accuracy of neurosurgical robotic systems and stereotactic frames. We develop a novel phantom (T-Phantom) which enables simultaneous localization of translational displacements in entry and target points. The phantom consists of multiple trajectories positioned around a localizer feature simulating approach trajectories in neurosurgical procedures on the intracranial space. Each trajectory consists of two parallel and coaxial hollow cylinders printed in selective laser sintering technology. We apply a stereo vision measuring method for precise measurements of translational displacements in target and entry positions. The paper further provides a systematic comparison of phantom designs originating from stereotactic frames and neurosurgical robotic systems. To the author's knowledge, the developed T-Phantom is the first stereotactic phantom which enables simultaneous measurements both in deviation from target and entry positions and angular deviation from the planned trajectory.
\end{abstract}

Keywords: Robotics; Neurosurgery: Stereotactic phantom: Stereotactic localization.

\section{Introduction}

The use of robotics in neurosurgery is experiencing constant growth and novel robotic systems are continuously being developed to assist in complex neurosurgery tasks. In particular, neurosurgery operations are extremely lengthy and tedious and the application of robots is expected to provide the surgeon and patient with multiple benefits [1], [2]. Neurosurgical procedures performed by a robotic system yield better and faster performance, are less invasive and enable faster recovery of the patient [3]. By this means the utilization of hospital operational resources is considerably improved. Prior to performing in vivo procedures, it is necessary to make a diverse range of testing on phantoms [1], [4] for testing the accuracy and consistency of robotic systems. The phantom representing a patient head (more specifically the intracranial space) does not necessarily need to be anthropomorphic. Phantoms developed for stereotactic procedures need to provide an accurate replica of targeted trajectories in stereotactic procedures. Phantom designs can also provide functionalities [5] for drilling procedures and tissue simulations with different characteristics of the human head (skin, bone, brain). This research discusses only the former problem, that of translational and angular displacements in 
trajectories. Each set of translational displacements can be further expressed as Euclidian distance errors. In the operating room these errors are accounted from CT or MRI scans, errors in registration procedures, mechanical errors in robotic systems or stereotactic frames and other.

In this paper we have developed a novel phantom called the T-Phantom (Trajectory Phantom) which is to the author's knowledge the first stereotactic phantom which enables simultaneous measurements both in deviation from target and entry positions and angular deviation from the planned trajectory. In the first part of the paper we give a systematic overview of various phantom designs for both robotic neurosurgical systems and for stereotactic frames. The problem of phantom design in neurosurgery has been tackled by many researchers. In each phantom design and associated accuracy measurement method we identify measurable variables and give a critical overview. Finally we discuss the design of the T-Phantom and apply a previously developed stereo vision measurement method [6] for objective accuracy measurement.

\section{Neurosurgical phantom designs}

The Neuromate (Renishaw) anthropomorphic phantom described in [7] (Fig. 1a) uses five implantable frameless markers (Fischer-Leibinger, Freiburg, Germany), randomly distributed on the surface, which are used for the registration in the infrared region. A ZD (Fischer-Leibinger, Freiburg, Germany) stereotactic frame is fitted onto the phantom. A different phantom design for the Neuromate robot [8] uses a technique of milling foam blocks (Fig. 1.b) to a predefined shape. Each of the foam blocks is measured after the milling procedure to acquire volumetric errors.

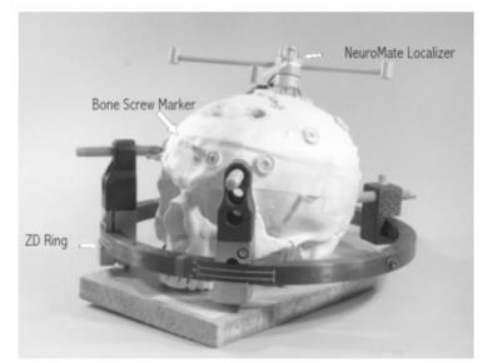

a)

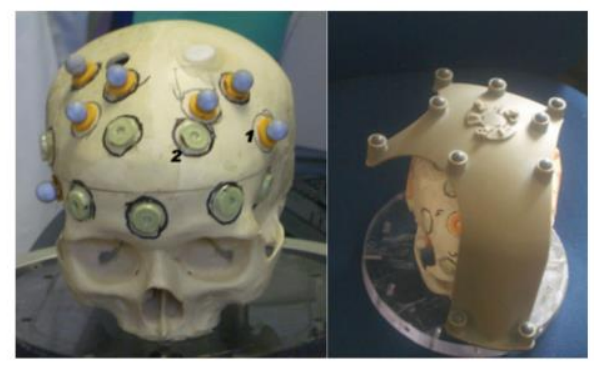

d)

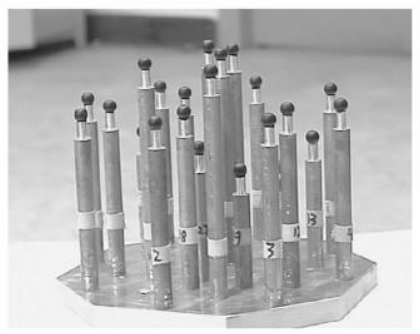

g)

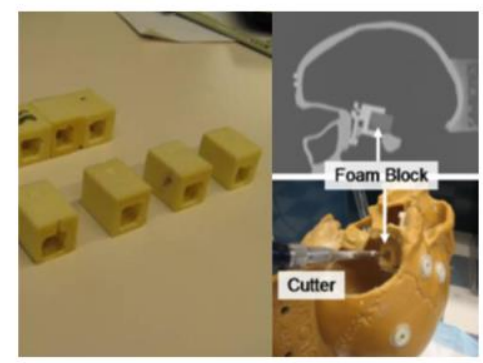

b)

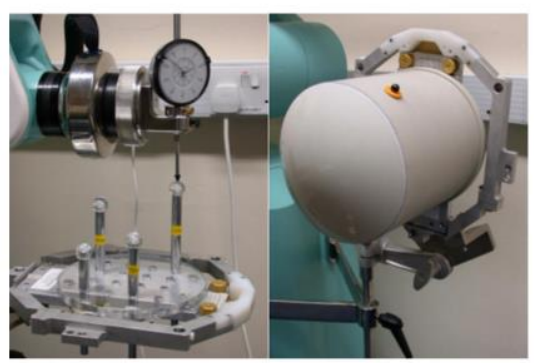

c)

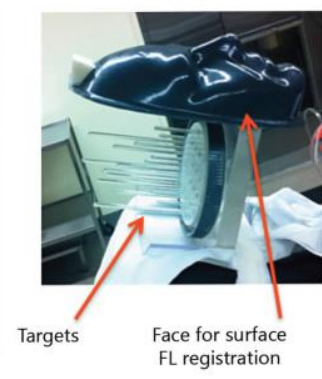

e)

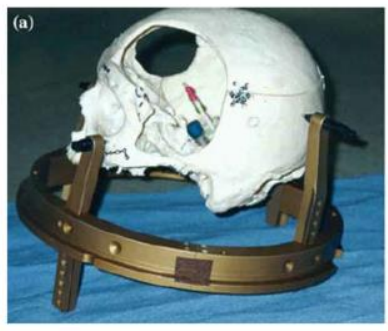

h)

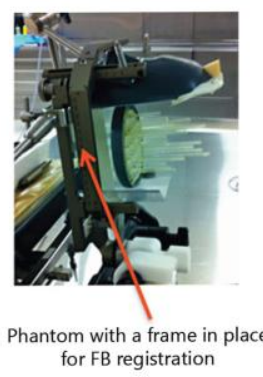
for $F B$ registration

(2)

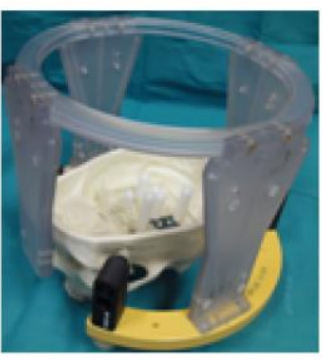

f)

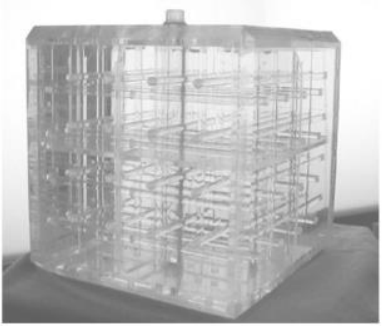

i)

Fig. 1. Diverse neurosurgical phantom designs: a) Neuromate phantom [7] b) Neuromate phantom [8] c) Pathfinder phantom [9] d) Pathfinder phantom [4] e) ROSA phantom [10] f) MARS phantom [11] g) NeuroMaster phantom [12]

h) CRW frame phantom [13] i) Leksell frame phantom [14]

The phantom used to check the robot system Pathfinder [9] consists of spherical targets (Fig. 1c) and detachable cylindrical surface serving as a simulation of skin to which the markers can be attached for the registration procedure. Spherical targets are $10 \mathrm{~mm}$ in diameter and are located at positions that simulate the most common target depths during neurosurgical operation procedures. A depth gauge is used to measure the application accuracy. Another phantom design for the Pathfinder robot [4] employs an anthropomorphic phantom. Onto a replica of the human skull registration markers 
and ten target surface points are mounted (Fig. 1d). The interior of the phantom is equipped with nine (9) depth target points. The measurements were made using vernier calipers.

The NeuroMaster [12] phantom (Fig. 1g) has a similar design and is made of cylinders with plastic spheres. Four spheres are used as markers for the registration of phantom while the other are used as target points.

The phantom [10] for the robotic system ROSA (Medtech innovative surgical technology) is shown in Fig. 1e. The phantom is made of a base with hollow polymer cylinders representing targets at different depths. A removable water tank gives a possibility of recording the phantom on MRI. The phantom has a hollow plastic face that can be filled with a contrast agent to make it suitable for scanning on MRI scanners.

The MARS stereotactic robot [11] uses commercially available phantoms made as replicas of the human skull (Fig. 1f). Inside the skull cylindrical conical cylinders represent target points. The measurement is performed with two vertically mounted cameras which measures the position of the probe tip deviation at the target point.

Phantom designs for stereotactic frames such as Leksell frame [14] and CRW frame [13] are shown in Fig. 1i and Fig. 1h. Other phantom designs include gel based phantoms [15] dedicated for MRI scanning, special phantoms with divots [16], hollow cylindrical phantoms [17] with different target materials and resin based phantoms [18] with implantable titanium screws.

\section{T-Phantom}

For the purpose of measuring the accuracy of robotic System RONNA [1] a phantom design called the T-Phantom is proposed. The T-Phantom (Fig. 2) consists of a Plexiglas construction and a localization plate (RONNAmarker) with three (or four) spherical markers. The spherical markers are used to define the phantom coordinate system. The phantom has hollow cylinders printed in selective laser sintering technology (SLS) which simulate operation trajectories.. The phantom is designed to simulate trajectories in neurosurgical applications under four tilt angles $\left(45^{\circ}, 35^{\circ}, 25^{\circ}, 15^{\circ}\right)$, and four trajectories perpendicular to the phantom base. The maximum spatial angle between two trajectories is $70^{\circ}$. Trajectories are selected in accordance with common trajectory angles in actual operations. The top of the T-Phantom has a radius simulating the top of the human head For attaching the localization plate (RONNAmarker). The phantom has machined grooves on each of the tilted trajectories so that the distance from entry to target point can be adjusted. Each trajectory consists of two parallel rectangular prisms with coaxial cylindrical bores. Three rings made from selective laser sintering (SLS) are located in each of the bores. The outer white cylinders are $2 \mathrm{~mm}$ thick and the inner black cylinder is $6 \mathrm{~mm}$ thick as shown in Fig. 3.

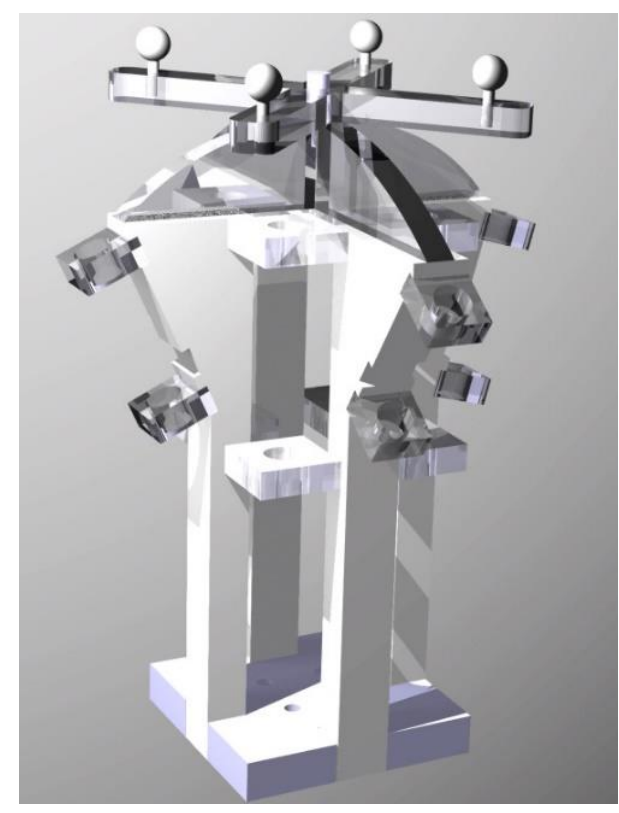

Fig. 2. a) T-phantom CAD prototype

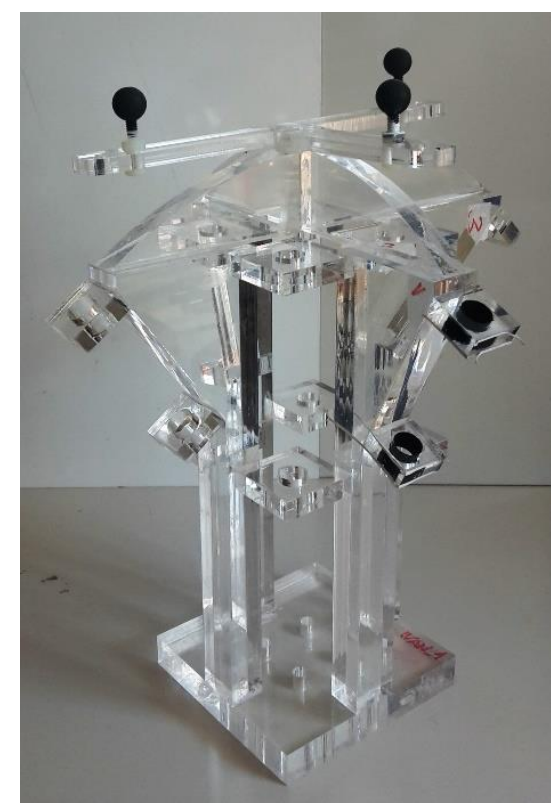

b)Actual T-Phantom

The measurement procedure is as follows. The T-Phantom is scanned on a CT scanner $(512 \times 512$ with $0,75 \mathrm{~mm}$ slice thickness, no gantry). The trajectories are planned in the operation planning software. The phantom is positioned in a Mayfield clamp after which the RONNA robotic system localizes the phantom and positions the tool guide to the planned trajectory. The surgical tool (probe) is calibrated to a predefined depth as the robot tool center point. The insertion of the probe is done manually to the predefined depth. At this step the measurement is carried out on the entry and target point for each trajectory as shown in from Fig. 3. 


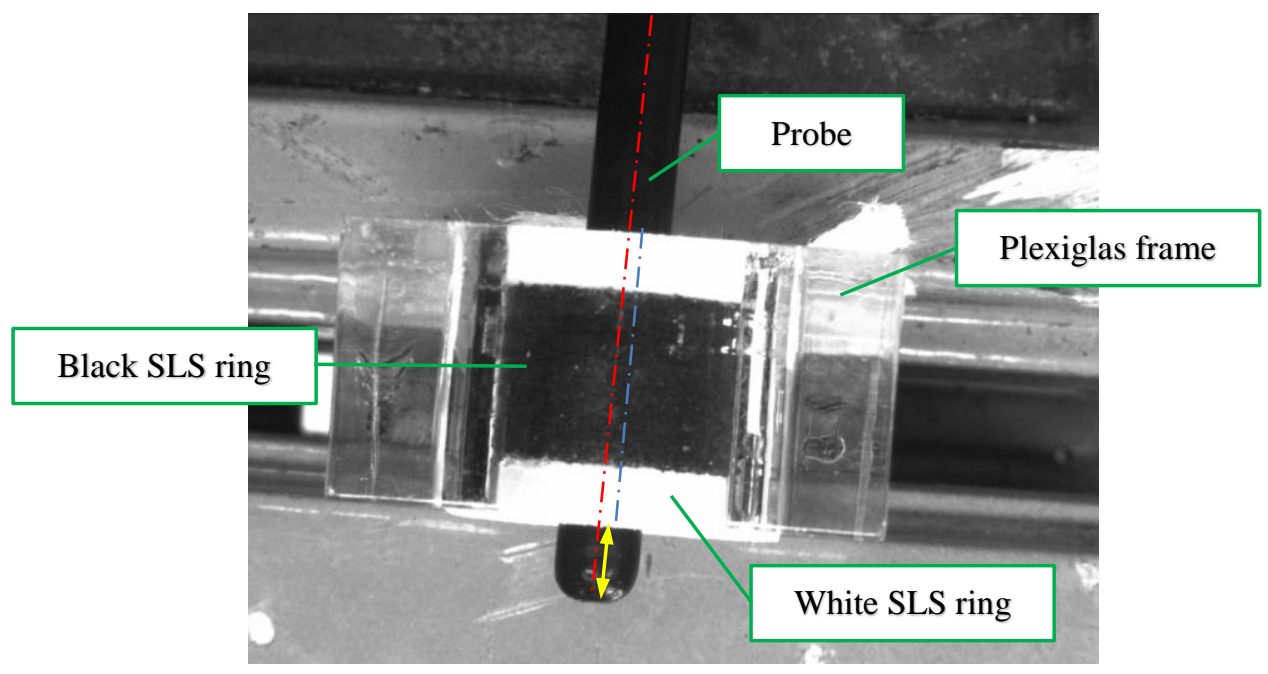

Fig. 3. a) Vision system measurement of the target point

In Fig. 3 the main measurement concept is depicted through a picture taken by our stereo vision system [6]. The picture shows a target point in which the depth error in the $\mathrm{Z}$ direction is depicted by the yellow arrow. The red dotted line represents the rotational axis of the probe while the blue dotted line represents the rotational axis of the cylindrical SLS inserts. By measuring the position of the probe relative to the phantom in two perpendicular camera directions the total Euclidian error can be computed for both the target and entry point. By measuring the XY translational error at the target point and the XY translational error of the Entry point the angular error is calculated. The angular error reflects the total (spatial) angular displacement error of the probe (red dotted line) with respect to the ideal trajectory (blue dotted line).

\section{Conclusion and future work}

Table 1. shows a detailed comparison of thirteen phantom designs and the new T-Phantom design. Both neurosurgical robotic phantoms and phantoms intended for stereotactic frames are compared (CRW Precision ${ }^{\mathrm{TM}}$ Arc system, ZD stereotactic system, Leksell Stereotactic System ${ }^{\circledR}$ ). The CT scan column (Computed Tomography) depicts if the phantom design is suitable for CT technology. MRI scan (Magnetic Resonance Imaging) depicts if the phantom design is suitable for MRI technology. Target point and entry point error define if it is possible to localize positioning errors of neurosurgical tools (probes, drills, etc.) with respect to planned operation points in a given phantom design. The angular error further takes into considerations errors derived from entry and target points. The last column in Table 1. differentiates anthropomorphic[5] phantoms from non-anthropomorphic phantom designs.

\begin{tabular}{|c|c|c|c|c|c|c|}
\hline Phantom (system) & $\begin{array}{c}\mathrm{CT} \\
\text { scan }\end{array}$ & $\begin{array}{l}\text { MRI } \\
\text { scan }\end{array}$ & \begin{tabular}{|c|} 
Target point \\
error
\end{tabular} & $\begin{array}{c}\text { Entry point } \\
\text { error }\end{array}$ & $\begin{array}{c}\text { Angular } \\
\text { error }\end{array}$ & $\begin{array}{c}\begin{array}{c}\text { Anthropomorphic } \\
\text { phantom }\end{array} \\
\end{array}$ \\
\hline Neuromate $\circledR[7]$ & yes & no & yes & no & no & yes \\
\hline Neuromate $\AA[8]$ & yes & no & yes & no & no & yes \\
\hline Pathfinder [9] & yes & no & yes & no & no & no \\
\hline Pathfinder [4] & yes & yes & yes & no & no & yes \\
\hline $\operatorname{ROSA}^{\mathrm{TM}}[10]$ & yes & yes & yes & yes & no & no \\
\hline MARS [11] & yes & yes & yes & no & no & yes \\
\hline NeuroMaster [12] & yes & no & yes & no & no & no \\
\hline CRW $^{\mathrm{TM}}$ frame [13] & no & yes & yes & no & no & yes \\
\hline Leksell@ frame [14] & yes & yes & yes & no & no & no \\
\hline Gel based phantom [15] & no & yes & yes & no & no & no \\
\hline Phantom with divots [16] & yes & yes & yes & no & no & no \\
\hline Cylindrical phantom [17] & yes & yes & yes & no & no & no \\
\hline Resin based phantom [18] & yes & no & yes & no & no & yes \\
\hline T-Phantom & yes & no & yes & yes & yes & no \\
\hline
\end{tabular}

Table 1. A systematic comparison of 13 phantom designs with the developed T-Phantom

It can be observed that only the ROSA ${ }^{\mathrm{TM}}$ phantom design enables simultaneous measurements of both errors in target and entry points. The ROSA phantom design with hollow cylinders does not enable objective quantitative measurements but only measurements of type "better than". The main advantage of the novel T-Phantom design is that it enables simultaneous objective measurements of translational errors in positioning of neurosurgical instruments at target and entry 
points. Our design also provides a possibility of angular displacement calculations and deviations from planned trajectories. The T-Phantom can be also used or adapted to other fields and applications where there is a need for measurements of deviations from planned translational (linear) trajectories. The T-Phantom design is scalable so that it can be used ranging from applications dedicated for microsurgery to applications where distances from target to entry point considerably exceed $120 \mathrm{~mm}$ (longest trajectories in neurosurgery).

In future research we plan to optimize the phantom design so that it can be machined from polymer components with higher accuracy. Also a new redesign should be done to enable scanning of the phantom using MRI technology. Furthermore, we plan to make a detailed accuracy analysis of the measurement method by comparing the stereo vision measurements with ground truth measurements made with either a coordinate measuring machine or a high accuracy vision system [19].

\section{Acknowledgments}

Authors would like to acknowledge the Croatian Scientific Foundation through the research project ACRON - A new concept of Applied Cognitive Robotics in clinical Neuroscience.

\section{References}

[1] B. Jerbić, G. Nikolić, D. Chudy, M. Švaco, and B. Šekoranja, "Robotic application in neurosurgery using intelligent visual and haptic interaction," International Journal of Simulation Modelling, vol. 14, no. 1, pp. 71-84, 2015

[2] G. R. Sutherland, S. Wolfsberger, S. Lama, and K. Zarei-nia, "The evolution of neuroArm," Neurosurgery, vol. 72 Suppl 1, pp. 27-32, doi: .1227/NEU.0b013e318270da19 2013

[3] M. Hoeckelmann, I. J. Rudas, P. Fiorini, F. Kirchner, and T. Haidegger, "Current Capabilities and Development Potential in Surgical Robotics," International Journal of Advanced Robotic Systems, p. 1, 2015

[4] M. S. Eljamel, "Validation of the PathFinder ${ }^{\mathrm{TM}}$ neurosurgical robot using a phantom," The International Journal of Medical Robotics and Computer Assisted Surgery, vol. 3, no. 4, pp. 372-377, doi: .1002/rcs.153 2007

[5] A. Müns, J. Meixensberger, and D. Lindner, "Evaluation of a novel phantom-based neurosurgical training system," Surgical Neurology International, vol. 5, no. 1, p. 173, 2014

[6] M. Švaco, B. Šekoranja, F. Šuligoj, and B. Jerbić, "Calibration of an Industrial Robot Using a Stereo Vision System," in Procedia Engineering, 2014, vol. 69, pp. 459-463

[7] Q. H. Li, L. Zamorano, A. Pandya, R. Perez, J. Gong, and F. Diaz, "The application accuracy of the NeuroMate robot--A quantitative comparison with frameless and frame-based surgical localization systems," Comput. Aided Surg., vol. 7, no. 2, pp. 90-98, doi: .1002/igs.10035 2002

[8] T. Haidegger, "Improving the Accuracy and Safety of a Robotic System for Neurosurgery," 2008

[9] P. S. Morgan, T. Carter, S. Davis, A. Sepehri, J. Punt, P. Byrne, A. Moody, and P. Finlay, "The application accuracy of the Pathfinder neurosurgical robot," International Congress Series, vol. 1256, pp. 561-567, Jun. 2003

[10] M. Lefranc, C. Capel, A. S. Pruvot, A. Fichten, C. Desenclos, P. Toussaint, D. Le Gars, and J. Peltier, “The Impact of the Reference Imaging Modality, Registration Method and Intraoperative Flat-Panel Computed Tomography on the Accuracy of the ROSA ${ }^{\circ}$ Stereotactic Robot," Stereotactic and Functional Neurosurgery, vol. 92, no. 4, pp. 242250, 2014

[11] M. Heinig, "Design and Evaluation of the Motor Assisted Robotic Stereotaxy System MARS,” Lubeck, 2012

[12] J. Liu, Y. Zhang, and Z. Li, "The application accuracy of neuromaster: a robot system for stereotactic neurosurgery," in Mechatronic and Embedded Systems and Applications, Proceedings of the 2nd IEEE/ASME International Conference on, 2006, pp. 1-5

[13] A. Quiñones-Hinojosa, M. L. Ware, N. Sanai, and M. W. McDermott, "Assessment of Image Guided Accuracy in a Skull Model: Comparison of Frameless Stereotaxy Techniques vs. Frame-Based Localization,” Journal of NeuroOncology, vol. 76, no. 1, pp. 65-70, Jan. 2006

[14] C. Yu, "An image fusion study of the geometric accuracy of magnetic resonance imaging with the Leksell stereotactic localization system," Journal of Applied Clinical Medical Physics, vol. 2, no. 1, p. 42, Jan. 2001

[15] A. D. Squires, Y. Gao, S. F. Taylor, M. Kent, and Z. T. H. Tse, "A Simple and Inexpensive Stereotactic Guidance Frame for MRI-Guided Brain Biopsy in Canines," Journal of Medical Engineering, vol. 2014, pp. 1-7, 2014

[16] D. Á. Nagy, T. Haidegger, and Z. Yaniv, "A Framework for Semi-Automatic Fiducial Localization in Volumetric Images," in Augmented Environments for Computer-Assisted Interventions, Springer, 2014, pp. 138-148

[17] S. Poggi, S. Pallotta, S. Russo, P. Gallina, A. Torresin, and M. Bucciolini, "Neuronavigation accuracy dependence on CT and MR imaging parameters: a phantom-based study," Physics in medicine and biology, vol. 48, no. 14, p. 2199, 2003

[18] G. Eggers and J. Muhling, "Template-based registration for image-guided skull base surgery," Otolaryngology Head and Neck Surgery, vol. 136, no. 6, pp. 907-913, Jun. 2007

[19] F. Suligoj, B. Jerbic, M. Švaco, B. Sekoranja, D. Mihalinec, and J. Vidakovic, "Medical applicability of a low-cost industrial robot arm guided with an optical tracking system," 2015, pp. 3785-3790 